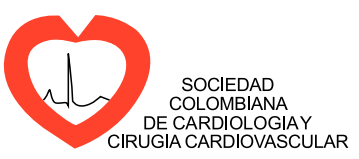

\title{
EDITORIAL
}

\section{¿Somos conscientes de una nueva nomenclatura de aterosclerosis?}

\section{Are we aware of a new atherosclerosis nomenclature?}

\author{
Darío Echeverri ${ }^{\mathrm{a}, \mathrm{b}, \mathrm{c}}$
}

\author{
a Servicio de Hemodinamia e Intervencionismo Cardiovascular, Fundación Cardioinfantil - Instituto de Cardiología, Bogotá, \\ Colombia \\ ${ }^{\mathrm{b}}$ Laboratorio de Investigación en Función Vascular, Fundación Cardioinfantil - Instituto de Cardiología, Bogotá, Colombia \\ c Facultad de Medicina, Universidad del Rosario, Bogotá, Colombia
}

Recibido el 26 de mayo de 2017; aceptado el 31 de mayo de 2017

Disponible en Internet el 27 de junio de 2017

La enfermedad arterial coronaria sigue siendo la principal causa de morbilidad y mortalidad en todo el mundo, pese a los muchos y continuos avances en los últimos años en programas de prevención y terapias médicas farmacológicas y de revascularización miocárdica. Aunque la supervivencia de los pacientes con enfermedad arterial coronaria ha ido mejorando, las enfermedades cardiovasculares representaron 17,3 millones de muertes en todo el mundo en 2012, y se espera que este número aumente a más de 23,6 millones en $2030^{1}$. Estimaciones recientes muestran que un tercio de los adultos en los Estados Unidos (aproximadamente 71,3 millones) tienen alguna forma de enfermedad cardiovascular, incluyendo cerca de 17 millones con enfermedad arterial coronaria y 10 millones con angina de pecho ${ }^{2,3}$.

La nomenclatura de las enfermedades degenerativas vasculares ha sido confusa y no ha existido unanimidad en los conceptos, hecho que refleja una ausencia de acuerdo entre los patólogos, y la propuesta de clasificaciones con deficiencias y que no unifican los fenómenos fisiopatológicos que se presentan. En general, ateroma, derivado de la palabra

\footnotetext{
Siguiendo la política de publicación de la revista, este artículo, en el que ha participado como coautor un miembro del Comité editorial, ha superado un estricto proceso de revisión por pares doble ciego y los autores no han tenido ninguna injerencia en la aprobación para su publicación.

Correo electrónico: decheverri@cardioinfantil.org
}

griega athere, hace alusión un material caseoso con alto contenido de lípidos en placas intimales avanzadas. Aterosclerosis, enfatiza el extenso componente esclerótico o de tejido fibroso en la lesión. En la literatura británica, ateroma y aterosclerosis con frecuencia son sinónimos. Arteriosclerosis, literalmente significa esclerosis o endurecimiento de las arterias como manifestación de la edad, independientemente de la presencia de aterosclerosis. En general, la arteriosclerosis es un concepto usado en forma genérica, e incluye aterosclerosis, esclerosis de Mönckkeberg y cambios relacionados en arterias periféricas grandes y pequeñas inducidos por la edad. La arterioloesclerosis, se refiere al engrosamiento, la fibrosis y la hialinización de las paredes de arteriolas con estrechamiento del lumen. La flebosclerosis, literalmente significa esclerosis o fibrosis de venas.

Desde la clasificación de la aterosclerosis descrita por Stary et al. ${ }^{4}$ hace más de 20 años, que fue aceptada por la comunidad científica y en especial por la Asociación Americana del Corazón (AHA, su sigla en inglés), ha habido importantes avances en diagnóstico, tratamiento farmacológico e intervenciones percutáneas y quirúrgicas que han influido notablemente en la enfermedad, y que han obligado a tener en cuenta una nueva nomenclatura y términos nuevos en la práctica clínica. Además de la aterosclerosis en vasos nativos, consistente en el proceso patológico en un entorno natural, dos de las nuevas patologías son el resultado de la formación de ateroma en un "entorno iatrogénico"; uno es la aterosclerosis de los injertos de vena 
Tabla 1 Nomenclatura de la aterosclerosis

\begin{tabular}{ll}
\hline 1 & Aterosclerosis de vasos nativos \\
& Incluye arteriosclerosis y arteriolosclerosis \\
2 & Aterosclerosis de injertos de vena safena \\
3 & Neoaterosclerosis \\
\hline
\end{tabular}

safena y otro la neoaterosclerosis en segmentos intervenidos previamente con stents. Así, se conforman varias entidades claramente diferentes a las cuales, hoy en día, tenemos que enfrentar (tabla 1).

\section{Aterosclerosis en arterias coronarias nativas}

A finales de los años setenta, Ross destacó la importancia de la proliferación de células de músculo liso en la formación de lesiones ateroscleróticas, y la hipótesis acerca de que la lesión de la pared arterial tiene un papel proponderante en la progresión de la placa. Posteriormente, se identificó la inflamación como la principal fuerza impulsora de la activación y proliferación de células de músculo liso, procesos mediados por factores de crecimiento. Estudios subsecuentes a finales de los años noventa, indicaron que el mecanismo de progresión de la enfermedad implicaba una interacción compleja entre los factores de riesgo y la inflamación. Durante el mismo período, Fuster et al. observaron la progresión de la placa como un fenómeno de eventos escalonados e identificaron que las fisuras y ulceraciones profundas de la placa resultaban en la manifestación de lesiones complejas, como causa de trombosis luminal y presentación clínica del síndrome coronario agudo.

- En la década de 1990 se publicó un documento de consenso de la AHA sobre la clasificación de la aterosclerosis ${ }^{4}$. La clasificación incluyó seis categorías distintas:

- Tipo ı, engrosamiento intimal.

- Tipo II, estría grasa.

- Tipo III, lesión transitoria o intermedia.

- Tipo Iv, ateroma avanzado con un núcleo necrótico bien definido.

- Tipo v, fibro-ateroma o ateroma con nuevo abundante tejido conectivo fibroso superpuesto.

- Tipo vi, placas complicadas con soluciones de continuidad (rupturas) en la superficie, hematoma o hemorragia, trombosis o una combinación de estas características.

Sin embargo, esta clasificación no lograba captar dos etiologías clínicas importantes en la formación del trombo coronario agudo, que difieren de la rotura de la placa, como son la erosión superficial, que representa el 25-30\% de los casos de trombosis y los nódulos calcificados eruptivos menos frecuentes, pero aún importantes, y que se presentan en menos del $5 \%$ de los pacientes. Además, a mediados de los años ochenta, Davies había identificado lesiones activas caracterizadas por fisuras de la placa como otra forma de comunicación entre el lumen y el núcleo necrótico subyacente ${ }^{5}$, que no estaba incluido en la clasificación de la AHA.

Con los avances en los biomarcadores e imágenes intracoronarias de las placas ateroscleróticas, el concepto de "placa vulnerable" como precursor de ruptura, tampoco encajaba perfectamente en una clasificación numérica ordenada. Estas restricciones, por lo tanto, han llevado a desarrollar un esquema modificado ${ }^{6}$, en el que los tipos I-IV de las lesiones descritas en la Clasificación de la AHA fueron reemplazados por términos descriptivos: engrosamiento intimal adaptativo, xantoma intimal (estrías grasas), engrosamiento intimal patológico y fibro-ateroma. Así mismo, los fibro-ateromas han sido subcategorizados más recientemente en placas de fase "temprana" y "tardía", sobre la base de las características líticas y no líticas del núcleo necrótico ${ }^{7}$. Las categorías $A$ y $v$ de la AHA han sido descartadas porque no tuvieron en cuenta las tres etiologías (ruptura, erosión y nódulo calcificado) que dan lugar a trombosis coronaria. Las lesiones precursoras de la ruptura de la placa, originalmente conocidas como "placas vulnerables", se clasificaron como fibro-ateroma de capa delgada (TCFA). También se introdujeron términos adicionales para implicar la reparación arterial en presencia de las placas con trombosis episódica silenciosa (ruptura de la placa sanada) y las fisuras de la placa.

\section{Aterosclerosis en injertos de vena safena}

La cirugía de revascularización coronaria es el estándar de atención para pacientes con enfermedad multivaso o enfermedad de tronco principal izquierdo y con fracción de eyección reducida, como lo apoyan muchos estudios que muestran una reducción de la morbilidad y la mortalidad en relación con la intervención coronaria percutánea. De las opciones de injertos, los de injertos de arterias mamarias internas, tienen la mejor permeabilidad a largo plazo; sin embargo, sólo 5 a $10 \%$ de los pacientes reciben arterias mamarias internas bilaterales debido al aumento de la morbilidad perioperatoria, la mortalidad, la duración de la operación y el riesgo de problemas de herida esternal. Así mismo, de los injertos de vena safena, el $10-25 \%$ se ocluyen por trombosis un año después de la cirugía y el 1-2\% adicional se ocluyen cada año de 1 año a 5 años después de la cirugía. Además, el $4-5 \%$ se ocluye cada año entre los 6 a 10 años de postoperatorio, debido al desarrollo acelerado de la aterosclerosis ${ }^{8}$.

En comparación con las arterias coronarias nativas, los injertos de vena safena son más susceptibles a la aterosclerosis, y el desarrollo de esta patología ocurre en una escala de tiempo acelerada. En el primer año después de la implantación, todos los injertos venosos muestran un engrosamiento intimal y una respuesta adaptativa a la circulación sistémica. La hiperplasia íntima consiste en una distribución concéntrica de células de músculo liso, proteoglicanos y colágeno tipo III. El cambio aterosclerótico más temprano en injertos de vena safena se informa alrededor de un año y se caracteriza por la acumulación de células espumosas seguida por el desarrollo de núcleo necrótico, que se observa entre 2 y 5 años después de la cirugía. Los implantes de injertos de vena safena de más de 5 años a menudo muestran expansión del núcleo necrótico a través de hemorragias intraplaca 9 . Los eventos hemorrágicos podrían contribuir a la ruptura de la placa, y suelen ocurrir entre 5 y 10 años después de la cirugía. En 10 años, sólo $38-45 \%$ de los injertos de vena safena siguen siendo permeables ${ }^{10}$. 
Análisis histopatológicos de injertos de vena safena implantados 8,5 \pm 5,9 años, han mostrado engrosamiento de la íntima, característica que contribuye a una estenosis media de $34 \pm 15 \%{ }^{11}$. Se observaron lesiones con infiltración exclusiva de células espumosas (xantomas intimales 0 «estrías grasas») en el $18,8 \%$ de las secciones con un porcentaje de estenosis de $37 \pm 9 \%$. Se observaron lesiones fibro-ateromatosas con núcleos necróticos en el $11,9 \%$ de las secciones, con estenosis media de $46 \pm 17 \%$. Las complicaciones de la hemorragia intraplaca en un núcleo necrótico se evidenciaron en el 7,5\% de las secciones. La morfología de lesión menos frecuente de ruptura de placa y trombosis se apreció en el 6,3\% de las secciones, con estenosis media de $75 \pm 24 \%$.

El engrosamiento circunferencial intimal es una respuesta adaptativa temprana en todos los injertos de vena safena. Los xantomas o "estrías grasas", están típicamente presentes más allá de un año. Sin embargo, a diferencia de la enfermedad coronaria nativa, las lesiones precoces de injertos de vena safena expresan grados variables de apoptosis de macrófagos, lo que conlleva núcleos necróticos que causan expansión de la placa. Los depósitos de lípidos no están involucrados en este proceso. La aterosclerosis de injertos de vena safena típica es a menudo concéntrica y difusa, con una cubierta fibrosa menos bien definida que en la enfermedad coronaria nativa; la cápsula fibrótica parece frágil y vulnerable a la ruptura.

Los factores críticos que subyacen al crecimiento neointimal rápido en injertos de vena safena son el resultado de lesión endotelial y excesivo estiramiento mecánico de la vena bajo presión arterial. La arterialización con engrosamiento fibrointimal es un cambio adaptativo consistente, que ocurre en los implantes de injertos de vena safena menores a un año de edad, y generalmente se acompaña de un estrechamiento del área de la sección transversal menor al 50\%. Una mayor presión arterial sistólica y diastólica se considera predictor importante del engrosamiento neointimal severo y posible oclusión; otros factores incluyen el diámetro del injerto de vena safena, el injerto en la arteria coronaria derecha y las características de los lechos arteriales en los vasos intervenidos ${ }^{12}$.

Los factores de riesgo cardiovascular también se aplican a la enfermedad de injerto de vena safena. Existe una buena correlación entre el colesterol total y el desarrollo de aterosclerosis de injerto venoso. La terapia agresiva con estatinas y que alcanza los niveles de colesterol LDL en un rango de $93-97 \mathrm{mg} / \mathrm{dl}$, reduce significativamente la aterosclerosis de injertos de vena safena $(27 \%)^{13}$. La terapia con estatinas o el tratamiento con $\beta$-bloqueadores también ha demostrado ser eficaz para limitar la enfermedad de injertos de vena safena ${ }^{12}$. Además, se ha demostrado que la terapia antiplaquetaria reduce el fracaso del injerto inducido por trombosis, pero no la permeabilidad a largo plazo ${ }^{14}$.

\section{Neoaterosclerosis}

En la actualidad, la intervención coronaria percutánea con stents (stents desnudos o no medicados (BMS, su sigla en inglés) o stents liberadores de fármacos (DES, su sigla en inglés) es el procedimiento más realizado para el tratamiento de pacientes con enfermedad arterial coronaria sintomática. La cicatrización y reparación arterial retardada con una cobertura pobre de los struts del stent se reconoce como el sustrato primario para la trombosis del stent atribuida al DES. Sin embargo, se ha informado que la neoaterosclerosis dentro del segmento del stent sea otra complicación que se presenta tanto en aquellos de la primera generación como de la segunda generación de DES, lo que se traduce en la falla tardía del stent por restenosis o trombosis del stent inducida por ruptura de placa.

La introducción de los stents coronarios al armamentarium del tratamiento de la enfermedad arterial coronaria, hace más de dos décadas cambió radicalmente el esquema de tratamiento de la enfermedad coronaria en todo el mundo. Es el procedimiento más utilizado para el manejo de los pacientes con síndrome coronario agudo y angina estable con enfermedad coronaria severa. La evolución en la tecnología permite disponer de mejores sistemas de liberación del stent, plataformas metálicas con aleaciones ( $\mathrm{CoCr} / \mathrm{PtCr}$, CoNi, PtLr, 316L) que las hacen más inertes y con menor posibilidad de inflamación peristrut y respuestas alérgicas, manteniendo sus capacidades mecánicas de flexibilidad y fuerza radial. Estas nuevas plataformas metálicas permiten disponer de diseños con struts más delgados entre 60 y $120 \mu \mathrm{m}$. También, existe una importante evolución en los polímeros (durables o biodegradables) que recubren el stent para permitir la liberación del fármaco (la mayoría de la familia Limus; sirolimus, everolimus, zotarolimus, biolimus). Los estudios más recientes demuestran que los DES de las nuevas generaciones no solo tienen ventajas en comparación con los $\mathrm{BMS}$, sino con los DES de primera generación en cuanto a reducción significativa a largo plazo de la revascularización de la lesión intervenida (TLR; $5,0 \%$ vs. 8,6\%), trombosis definitiva de stents $(0,9 \% \text { vs. } 2,5 \%)^{15}$.

Otro de los avances importantes en la tecnología del intervencionismo cardiovascular, son los stents no metálicos biorreabsorbibles (del inglés scaffolds y de la sigla BVS). Están elaborados en materiales bioabsorbibles (ácido L-poliláctico - PLLA), no tóxicos y que se reabsorben completamente en 2 a 3 años del implante; también liberan "limus" y ofrecen un concepto novedoso para el tratamiento de la enfermedad aterosclerótica nativa. Los estudios comparativos entres DES y BVS han demostrado resultados similares en TLR pero mayor incidencia de trombosis definitiva y probable ${ }^{16}$. Por tanto, entre las limitaciones descritas hoy en día de los stents surgen la restenosis, la trombosis y ahora recientemente descrita, la neoaterosclerosis.

Esta última se define como la acumulación de macrófagos y células espumosas, en el interior de la neoíntima formada en el segmento con un stent, con o sin formación de núcleo necrótico, calcificación o trombosis subyacente ${ }^{17}$. Este proceso implica que a partir de una neoíntima formada (a diferencia de la aterosclerosis nativa), con un proceso de reendotelización retardado y una reducción en la actividad de las células de músculo liso con alteraciones en las uniones intercelulares, existe disfunción endotelial, alteraciones en el shear stress, activación celular a un fenotipo pro-inflamatorio, respuesta permanente a cuerpo extraño, neovascularización y reclutamiento de macrófagos. En cuanto a histología, se caracteriza por depósito de macrófagos y formación de células espumosas vivas y apoptóticas, formación de un núcleo lipídico estable, microcalcificaciones incipientes dentro de la íntima, placas vulnerables (TCFA), y hemorragias intraplaca. 
Está claro hoy en día, gracias a estudios de imágenes intravasculares y hallazgos histopatológicos, que la neoaterosclerosis en los DES, ocurre más pronto y hay mayor presencia de macrófagos y células espumosas, al gual que posibilidad de TCFA y riesgo de ruptura de placa, con posible trombosis del stent ${ }^{18}$.

La neoaterosclerosis es una entidad cuya prevalencia clínica aun es desconocida. Solo puede ser detectada mediante imágenes intravasculares (ultrasonido intravascular o tomografía de coherencia óptica-OCT). La OCT posee una resolución axial mayor hasta de $10-20 \mu \mathrm{m}$, que permite identificar en la neoíntima intrastent células espumosas y por ende neoaterosclerosis temprana ${ }^{19}$. Con base en los hallazgos de imágenes, se considera una prevalencia de la neoaterosclerosis en DES de primera generación con plaquitaxel del $21 \%$, con sirolimus del $37 \%$ y en DES de segunda generación liberadores de everolimus del $29 \%{ }^{20}$.

En la actualidad, existe gran interés en el proceso de la neoaterosclerosis como causa de trombosis del stent, y su posible relación con la aterosclerosis nativa subyacente. La neoaterosclerosis pone en evidencia una vez más las deficiencias en las estrategias en prevención secundaria.

En conclusión, la comprensión de los procesos de aterosclerosis nuevos y la comparación morfológica y de la fisiopatología de la progresión de la placa aterosclerótica, debería ayudar a avanzar en el entendimiento de la enfermedad aterosclerótica nativa, la aterosclerosis de injertos de vena safena y la neoaterosclerosis y, por consiguiente, en el desarrollo de estrategias mejoradas para la modificación del riesgo cardiovascular y la prevención secundaria de la enfermedad. El futuro cercano está dirigido a generar ideas y nuevas estrategias para la identificación y tratamiento de la aterosclerosis en todas sus formas, la innovación en plataformas con terapias antiateroscleróticas y el fortalecimiento de los programas de prevención secundaria.

\section{Bibliografía}

1. Laslett LJ, Alagona P Jr, Clark BA, Drozda JP Jr, Saldivar F, Wilson SR, et al. The worldwide environment of cardiovascular disease: prevalence, diagnosis, therapy, and policy issues: a report from the American College of Cardiology. J Am Coll Cardiol. 2012;60(Supp l):S1-49.

2. Fihn SD, Gardin JM, Abrams J, Berra K, Blankenship JC, Dallas AP, et al. American College of Cardiology Foundation/American Heart Association Task Force. 2012 ACCF/AHA/ACP/AATS/PCNA/SCAI/STS guideline for the diagnosis and management of patients with stable ischemic heart disease: a report of the American College of Cardiology Foundation/American Heart Association task force on practice guidelines, and the American College of Physicians, American Association for Thoracic Surgery. Preventive Cardiovascular Nurses Association, Society for Cardiovascular Angiography and Interventions, and Society of Thoracic Surgeons. Circulation. 2012;126:e354-471.

3. Benjamin EJ, Blaha MJ, Chiuve SE, Cushman M, Das SR, Deo R, et al. Heart Disease and Stroke Statistics-2017 Update: A Report From the American Heart Association. American Heart Association Statistics Committee and Stroke Statistics Subcommittee. Circulation. 2017;135:e146-603.

4. Stary HC, Chandler AB, Dinsmore RE, Fuster V, Glagov S, Insull W Jr, et al. A definition of advanced types of atherosclerotic lesions and a histological classification of atherosclerosis.
A report from the Committee on Vascular Lesions of the Council on Arteriosclerosis, American Heart Association. Arterioscler Thromb Vasc Biol. 1995;15:1512-31.

5. Davies MJ, Thomas A. Thrombosis and acute coronary-artery lesions in sudden cardiac ischemic death. N Engl J Med. 1984;310:1137-40.

6. Virmani R, Kolodgie FD, Burke AP, Farb A, Schwartz SM. Lessons from sudden coronary death: a comprehensive morphological classification scheme for atherosclerotic lesions. Arterioscler Thromb Vasc Biol. 2000;20:1262-75.

7. Kolodgie FD, Gold HK, Burke AP, Fowler DR, Kruth HS, Weber DK, et al. Intraplaque hemorrhage and progression of coronary atheroma. N Engl J Med. 2003;349:2316-25.

8. Bourassa MG, Fisher LD, Campeau L, Gillespie MJ, McConney M, Lespérance J. Long-term fate of bypass grafts: the Coronary Artery Surgery Study (CASS) and Montreal Heart Institute experiences. Circulation. 1985;72:V71-8.

9. Walts AE, Fishbein MC, Matloff JM. Thrombosed, ruptured atheromatous plaques in saphenous vein coronary artery bypass grafts: ten years' experience. Am Heart J. 1987;114: 718-23.

10. Loop FD, Lytle BW, Cosgrove DM, Stewart RW, Goormastic M, Williams GW, et al. Influence of the internal-mammary- artery graft on 10-year survival and other cardiac events. N Engl J Med. 1986;314:1-6.

11. Yazdani SK, Farb A, Nakano M, Vorpahl M, Ladich E, Finn AV, et al. Pathology of drug-eluting versus bare-metal stents in saphenous vein bypass graft lesions. JACC Cardiovasc Interv. 2012;5:666-74.

12. Atkinson JB, Forman MB, Vaughn WK, Robinowitz M, McAllister $H A$, Virmani R. Morphologic changes in long-term saphenous vein bypass grafts. Chest. 1985;88:341-8.

13. Post Coronary Artery Bypass Graft Trial Investigators. The effect of aggressive lowering of low-density lipoprotein cholesterol levels and low-dose anticoagulation on obstructive changes in saphenous- vein coronary-artery bypass grafts. N Engl J Med. 1997;336:153-62.

14. Harskamp RE, Lopes RD, Baisden CE, de Winter RJ, Alexander $\mathrm{JH}$. Saphenous vein graft failure after coronary artery bypass surgery: pathophysiology, management, and future directions. Ann Surg. 2013;257:824-33.

15. Piccolo R, Pilgrim T, Heg D, Franzone A, Rat-Wirtzler J, Räber $L$, et al. Comparative effectiveness and safety of new generation versus early-generation drug-eluting stents according to complexity of coronary artery disease. J Am Coll Cardiol Int. 2015;8:1657-66.

16. Cassese S, Byrne RA, Ndrepepa G, Kufner S, Wiebe J, Repp J. Everolimus-eluting bioresorbable vascular scaffolds versus everolimus-eluting stents: a meta-analysis of randomized controlled trials. The Lancet. 2016;387:537-44.

17. Otsuka F, Byrne RA, Yahagi K, Mori H, Ladich E, Fowler DR, et al. Neoatherosclerosis: overview of histopathologic findings and implications for intravascular imaging assessment. Eur Heart J. 2015;36:2147-59.

18. Nakazawa G, Otsuka F, Nakano M, Vorpahl M, Yazdani SK, Ladich $\mathrm{E}$, et al. The pathology of neoatherosclerosis in human coronary implants bare-metal and drug-eluting stents. J Am Coll Cardiol. 2011;57:1314-22.

19. Malle C, Tada T, Steigerwald K, Ughi GJ, Schuster T, Nakano M, et al. Tissue characterization after drug-eluting stent implantation using optical coherence tomography. Arterioscler Thromb Vasc Biol. 2013;33:1376-83.

20. Otsuka F, Vorpahl M, Nakano M, Foerst J, Newell JB, Sakakura K, et al. Pathology of second generation everolimus-eluting stents versus first-generation sirolimus and paclitaxel-eluting stents in humans. Circulation. 2014;129:211-23. 